\title{
New Directions in RF Sources *
}

\author{
Levi Schächter \\ Laboratory of Plasma Studies and School of Electrical Engineering \\ Cornell University, Ithaca, NY 14853 USA
}

\section{Abstract}

We examine the various high power $R F$ sources on the basis of the next linear collider requirements. The discussion is based on classification of the sources according to what we consider the most informative criteria. In the conventional acceleration scheme (e.g. SLAC) multiple sources generate the RF power. A variety of sources are considered and they cover the spectrum from 9 to $19 \mathrm{GHz}$. In the two beam accelerator scheme all the modules are driven by the same beam which has multi $M e V$ (LBL/LLNL) up to a few GeV (CERN) electrons; the current carried by the beam is of order of $k A$. Two types of RF sources have been suggested: traveling wave (TW) structures and free electron laser (FEL). The operating frequency varies from device to device in the range of 9 to $35 G \mathrm{Cz}$.

\section{INTRODUCTION}

The typical requirements from an RF source for the next linear collider (NLC) are: $200 \mathrm{MW} / \mathrm{m}$ at $10-30 \mathrm{GHz}$ with an efficiency above $40 \%$. The pulse duration varies from one scheme to another, but the net pulse for acceleration is expected to be above $100 \mathrm{nsec}$ and to generate a gradient on the order of $100 \mathrm{MV} / \mathrm{m}$. The luminosity requirements determine the repetition rate and according to the system it can vary from $50-1400 \mathrm{~Hz}^{(1)}$. The detailed specifications of any RF source are established as part of the design of an entire system. According to the acceleration system one can divide these sources into two groups: sources which drive (i) a Two Beam Accelerator (TBA) e.g. at LBL/LLNL/MIT, KEK, JINR(Dubna) and CERN in which a single beam generates the whole required power and (ii) a conventional multiple sources system e.g. SLAC, KEK, DESY, VLEPP, Cornell and Univ. of Maryland; see Fig. 1. This is our first classification criterion.

If in the past the klystron was practically the only RF source used for acceleration purposes, the beam-wave interaction in today's systems tends to expand beyond the limits of a fraction of the (vacuum) wavelength. The main reason for this trend is the breakdown problem associated with the high power, frequency and efficiency imposed by the NLC requirements. The approach followed to overcome the breakdown problem provides our second classification criterion. There are several such approaches. (i) It is possible to increase the frequency (to $30 \mathrm{GHz}$ and above as is the case for the TBA); doing so the power level required is much smaller since for the same gradient the power scales like $f^{-2}$. Thus an increase by a factor of 3 in the frequency lowers the power requirement by almost an order of magnitude. However a problem with this approach is that the geometrical (alignment and manufacturing) tolerances at small dimensions, associaled with the increased frequency, become very tight $(0.5 \mu \mathrm{m})$. (ii) Another approach is that of pulse compression adopted by SL $\Lambda \mathrm{C}$ and KEK in which an $X$-band (11.4GHz) klystron generates $50 \mathrm{MW}$ for $1.5 \mu \mathrm{sec}$. This pulse is then compressed 4 times in power and 6 times in time such that the nominal pulse is $200 \mathrm{MW}$ for $250 \mathrm{nsec}$. A similar approach was adopted at VLEPP which operates at $14 G \mathrm{~Hz}$ and the compression method is different; we shall discuss this system later in more detail. (iii) The last approach to overcome the breakdown problem is to use a distributed interaction in a TW structure. This approach has proven to be successful at SLAC ${ }^{(2)}$, Cornell ${ }^{(3)}$ and with the Choppertron ${ }^{(4)}$ (Haimson/LLNL). The idea here is to generate a high power pulse $(100-400 \mathrm{MW})$ for the duration required for acceleration ( $>100 \mathrm{nsec}$ ). TW structures are also part of the CLIC extraction section.

The issue of distributed interaction leads us to the third classification criterion namely, we would like to distinguish here between (i) slow wave devices (Cerenkov) and (ii) fast wave devices (FEL, CARM ${ }^{(5)}$, Gyrotron ${ }^{(6-7)}$ and Magnicon $\left.{ }^{(8-9)}\right)$. This is schematically presented in Fig. 2. Each of these categories has its own advantages and disadvantages. If we limit for a moment the discussion to traveling wave amplifiers (TWA) and gyrotrons then the former operates in a single TM mode whereas in the latter a massive higher mode suppression is necessary for "single mode operation". On the other hand, in the TWA the breakdown at the corrugated walls remains a concern (though not as severe as in the klystron) whereas in a $\mathrm{gy}$ rotron the smooth walls minimize this problem. As for now it is difficult to assess what weight to attribute to the cost of the applied magnetic field and its required accuracy ${ }^{(7)}$ since the power levels reported so far are almost one order of magnitude lower than the $\mathrm{TWA}^{(10)}$. For $27 M W$ at $9.85 \mathrm{GHz}$ a field less than $6 \mathrm{kG}$ was required with an accuracy of $50 G$. This magnitude is similar to that of the guiding field in either TWA or relativistic klystron but these two are practically insensitive to variations in the magnetic field - on the scale mentioned above. We shall now examine some of the R.F sources which have been investigated recently in the view of the classification mentioned above.

\section{TWO BEAM ACCELERATOR}

The TBA concept first suggested in 1982 by A. Sessler (11) relies on the interaction of a single high power beam with a radiation field in the presence of a wiggler. The radiation power is extracted from discrete (extended) cavities, thus 


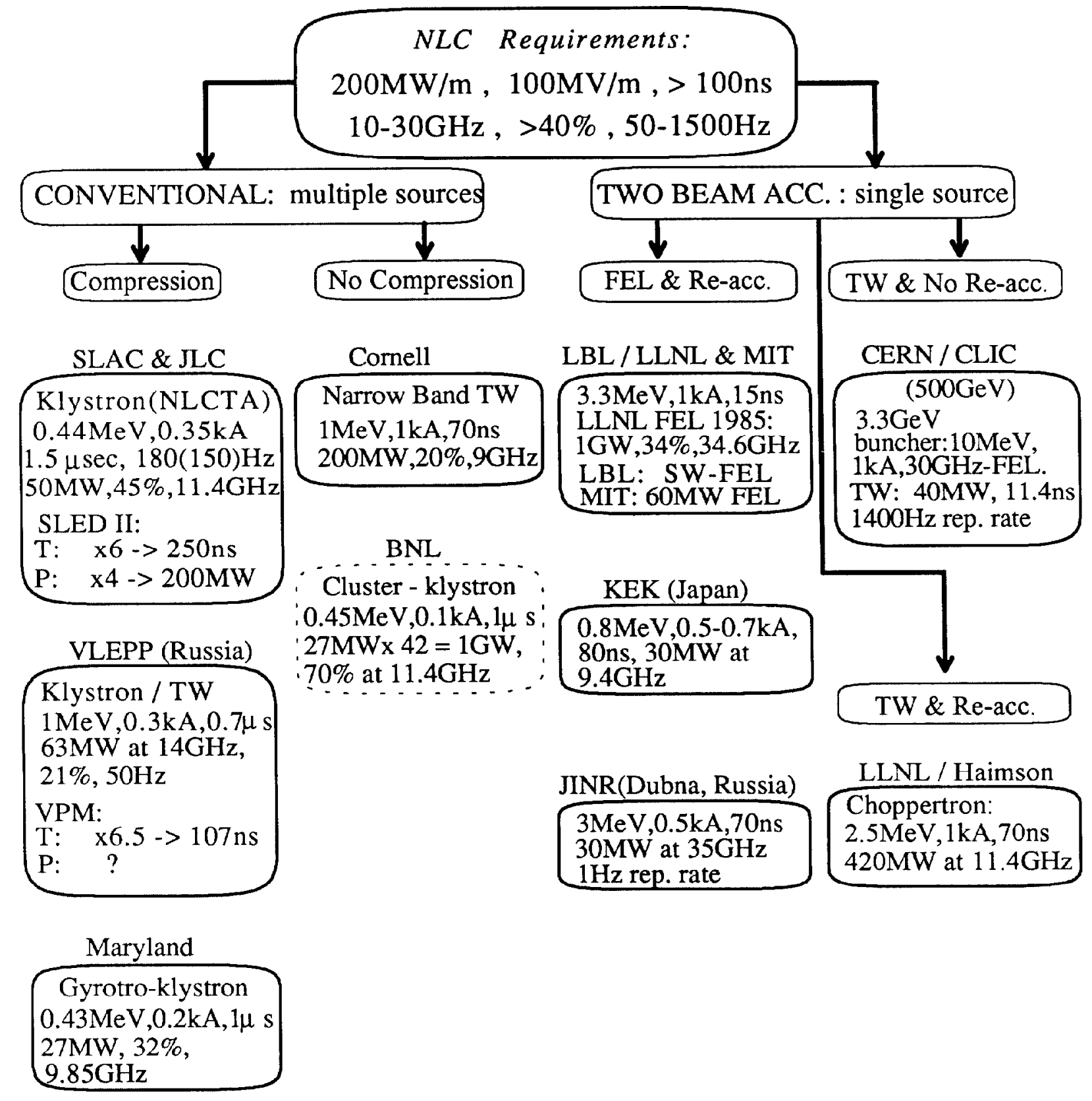

Fig.1: Classification of RF sources according to acceleration scheme. 
the notion of standing wave FEL. The proof of principle dates back to 1985 when a $3.3 \mathrm{MeV}, 1 k \mathrm{~A}$ beam generated $1 \mathrm{GW}$ of power for $15 \mathrm{nsec}$ at $34.6 \mathrm{GHz}$ corresponding to $34 \%$ efficiency ${ }^{(12)}$. Recently $30 M W$ at $9.4 G \mathrm{~Hz}$ were generated at $\mathrm{KEK}^{(13)}$ for $80 \mathrm{nsec}$ with $0.8 \mathrm{MeV}, 0.5-0.7 \mathrm{kA}$ in an FEL. At JINR(Dubna) $)^{(14)}$, a similar amount of power was generated for $70 \mathrm{nsec}$ but at $35 \mathrm{GHz}$ with a $3 \mathrm{MeV}, 0.5 \mathrm{kA}$ beam. All three have the FEL interaction in common. The beam is planned to be re-accelerated by induction linac cells which are crucial for the adequate operation of the following RF extraction units. According to our third classification the standing wave FEL is a fast wave device. There is no reason why the fast-wave interacting cells can not be replaced with slow-wave type cells. This is the case in the LLNL/Haimson ${ }^{(4)}$ experiment (Choppertron) in which a $2.5 \mathrm{MeV}, 1 \mathrm{kA}, 70 \mathrm{nsec}$ beam is injected in two consecutive traveling wave structures; power in excess of $400 \mathrm{MW}$ at $11.4 \mathrm{GH} z$ was measured in this case. Similar structures are used at CLIC following a very unique design. The electrons are bunched by an FEL when injected with an initial energy of $10 \mathrm{MeV}$ ( $1 \mathrm{kA}$ of current). They are bunched at $30 \mathrm{GHz}$ in this stage and then accelerated to $3.3 \mathrm{GeV}$. This beam feeds short traveling wave sections which generate about $40 M U$ of power, each for $11.4 n \mathrm{sec}$. Note that the $40 \mathrm{MW}$ in this design can generate a similar gradient as the $400 \mathrm{MW}$ of the Choppertron at $11.4 \mathrm{GHz}$.

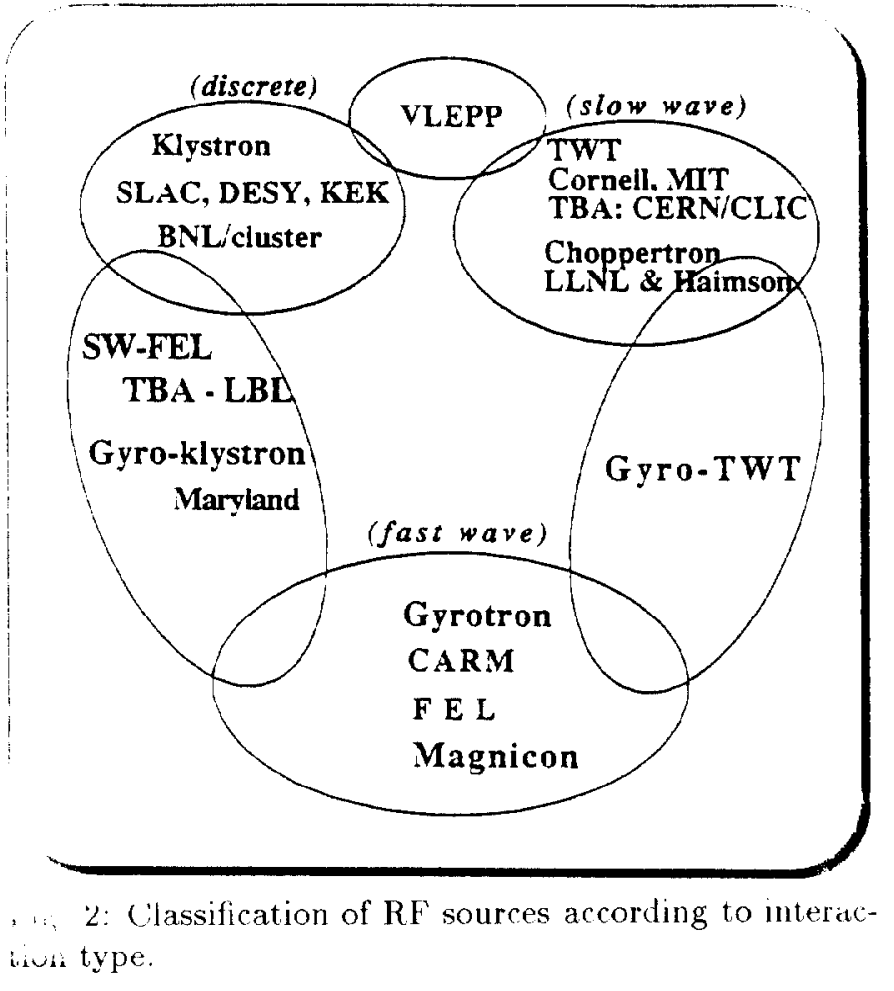

\section{CONVENTIONAL SOURCES}

When examining conventional sources it is convenient to divide them according to the interaction type. We shall discuss first the fast wave devices. In this presentation we shall limit the discussion to a single representative from this family namely, the gyrotron. The gyrotron ${ }^{(6-7)}$ or its combination with TW and klystron version called gyrotwystron has a significant appeal since the 'IW structure helps to keep the magnetic field at relatively low levels when the RF power levels (and thus the current) are significantly higher. The operation of the extraction section designed to fit the second frequency harmonic is also in progress.

In the category of the bare klystron we would like to mention the Cluster Klystron. As presently envisaged it is to be driven by a $0.45 \mathrm{MeV}, 0.1 \mathrm{kA}, 1 \mu \mathrm{sec}$ beam. There will be 42 such beams generated on the same cathode by magnetron injector guns which will generate beams with a local current density as high as $40 \mathrm{~A} / \mathrm{cm}^{2}$. At about $70 \%$ efficiency each klystron will generate $26 \mathrm{MW}$ with an overall $1.1 G W \mathrm{RF}$ power at $11.4 \mathrm{GHz}$; this is the first system discussed so far that has not been tested experimentally. Basically there are two major obstacles this system has to overcome: the generation of controllable multiple beams from a single cathode and, assuming that this was accomplished, the combination of all outputs in a single waveguide at a well defined phase.

As illustrated in Fig.2 the VLEPP klystron lies somewhere between the slow wave devices and the klystron fanily. It consists of a series of cavities but its extraction section consists of a traveling wave section. Its injection section is unique: the electrons are generated and guided by a $25 \mathrm{kV}$ electrostatic optic system and afterwards accelerated by a $1 M V d c$ voltage source. Since the driving voltage is high, the required power can be supplied by injecting a modest amount of current $(300 \mathrm{~A})$. This fact has two implications: (i) the beam can be guided by a permanent periodic magnetic field and (ii) according to $\mathrm{V}$. Balakin $^{(15)}$ it has the potential of a very efficient device due to its low (0.3) micro-perveance. The dc guiding of the beam makes the device very attractive from the financial point of view since a significant portion of the cost of an RF generator is the cost of the magnets. The design of this system is very ambitious and it is planned to reach almost $80 \%$ efficiency. At the present time the output is $63 \mathrm{MW}$ which corresponds to $21 \%$ efficiency. This is the typical efficiency one can expect from an uniform TW section without any optimization.

The last subject to be considered in the category of conventional sources is the traveling wave amplifier (TWA). We have investigated this device at Cornell both experimentally and theoretically in the past several years. In a two stage TWA we have reported generation of more than $400 M W$ in the $\mathrm{X}$-band for a pulse duration of $70 \mathrm{nsec}$ using a $1 M V, 1 k A$ beam. However the spectrum of the signal was unacceptably broad $(300 \mathrm{M} \mathrm{Hz})$ due to the presence of asymmetric sidebands. Theoretical studies indicated that these effects are a result of amplified noise at frequencies which are selected by the interference of the two waves which bounce between the two ends of the TW 
structure. In order to eliminate the problem of the reflections (the increased noise is unavoidably part of the modulation process) we suggested ${ }^{(16)}$ building an amplifier in which the time it takes the EM energy to reach the input end after being reflected from the output end, is of the same order of magnitude as the electron pulse duration. In order to satisfy the above condition the group velocity has to be very small (in our case less than $0.01 \mathrm{c}$ ). This subject is presented in Ref.10. The output in the most recent experiments indicate power levels of about $200 \mathrm{MW}$ at $9 \mathrm{GH} z$ in a frequency range which is less than $50 \mathrm{MHz}$. This has been achieved in an uniform structure in which the only optimization was making the last disk $1 \mathrm{~mm}$ thick instead of $6 \mathrm{~mm}$. This allows the EM mode to leave the system since for the disk radius this wave is well below cutof ${ }^{(18)}$. This result can be probably improved significantly by a better design of the output TW section.

\section{DISCUSSION}

Although the variety of concepts and devices is not particularly helpful in the attempt to identify a global trend, we believe that there are clear indications of local irends. The clearer among these trends is that of the TBA source: neither of the devices under consideration use the klystron as a basis for their RF generator. In fact it is fairly clear that the distributed interaction either in a fast or slow wave device replaces the local beam-wave interaction of a klystron. However the cavity effects, say in a standing wave FEL, are still there, and as was indicated in Ref.(17) the analogy to klystrons can be quite helpful.

With respect to conventional schemes, the scaling of the klystron to the X-band and to long pulse operation in conjunction with the compression scheme indicates that the klystron is still a potentially viable competitor for the next linear collider RF source. As an intermediary stage the compression schemes proposed by SLAC and VLEPP will probably meet the requirements but they will not solve the long term problem. Over the long term we have to remember the limitations of both the klystron and the compression schemes - especially with respect to breakdown. One of the alternatives we mentioned in our second classification criterion was a medium length (100$200 n s e c)$ high power $(100-400 M W)$ pulse. This kind of system eliminates the neccessity for compression and still provides the required power levels. But to withstand high electric field associated with these power levels it will require a TW output section ${ }^{(2-4)}$. Since this scheme has the potential to solve the problem it seems probable that TW output sections will become an integral part of future $\mathrm{RF}$ extraction systems. It is this trend which we wish to further discuss next.

The starting point is the constraint imposed by the NLC requirements on the $\mathrm{RF}$ structure. The output frequency requirement limits the input section of any source to a very good frequency selective device. From this perspective the klystron cavity or a combination of a cavity with a magnetic field as in the case of the Choppertron or an
FEL, are the natural candidates for the input section of any RF system. The main section can be a set of isolated cavities as in a klystron, a TW section or a combination of the two. However the breakdown problem will force us to use a TW structure as an output section with one or more extraction ports. This brings us to the last subject which is discussed in more detail in another work ${ }^{(18)}$ regarding the beam-wave interaction in quasi-periodic structures.

A high power traveling wave structure is conceived as a section of a periodic disk loaded structure and its electromagnetic characteristics are determined as if the system was infinitely long. Practically these are a set of cavities which are coupled through the disk aperture. At the other extreme, the klystron is a set of a few isolated cavities. In the former case the beam interacts with a wave continuously, whereas in the klystron the beam interacts with the field in the close vicinity of the cavity. The interaction in a uniform periodic structure (TWA) or in a few uncoupled cavities (klystron) is relatively well understood. But we lack analytical or even quasi-analytical tools to accurately investigate the interaction in transition region of a high power microwave device - which is exactly what is required for construction of an adequate output section. For this purpose we have developed an analytical method to investigate the beam-wave interaction in a quasi-periodic structure. The method relies on an arbitrary number of pill-box like cavities of any dimension and an arbitrary number of radial arms. The only constraint is that the radius of the coupling pipe has to be fixed. So far we have successfully employed this method for cold design in particular for the extraction region of the two stage amplifier reported in Ref.(10).

\section{SUMMARY}

Let us now summarize the main conclusions regarding high power radiation sources for acceleration applications: (i) 'There is a clear advantage to operate at the highest frequency the alignment constraints permit. (ii) At very high power local beam-wave interaction (as in a klystron) is disadvantageous comparing to distributed interaction in traveling wave structures due to the breakdown problem. (iii) Radiation sources need to expand further beyond the limits of the bare klystron. (iv) The expansion so far is "equally" divided towards fast and slow wave devices. (v) In two beam accelerators the klystron, in its present configuration, will play little, if any, role. (vi) In conventional sources the traveling wave structure with one or multiple output ports will have an increasing role at least in the output section of a high power RF source. Accordingly the interaction in transition regions will require more and more attention.

\section{ACKNOWLEDGEMENT}

I would like to thank J.A. Nation for his help in gathering the information and for the clarifying discussions at various stages of this work. 


\section{REFERENCES}

[*] This work was supported by the United States Department of Energy.

[1] There is no world wide consensus regarding the exact specification of the source for the next linear collider therefore we shall consider the figures above as figures of merit.

[2] "High-gradient electron accelerator powered by a relativistic klystron", M.A. Allen et. al. Phys. Rev. Lett. 63 p.2472 (1989).

[3] "Two Stage High Power Traveling Wave Amplifier" D. Shiffler, J. D. Ivers, G. S. Kerslick, J. A. Nation and L. Schächter, Appl. Phys. Lett., 58, 899, (1991).

[4] Suppression of beam induced pulse shortening modes in high power RF generator TW output structures", J. Haimson et. al. SPIE Proc. Vol.1629 p.209 (1992).

[5] "CARM Amplifier experiment at $17 \mathrm{GHz}$ " W. Menninger, B. Danley, S. Allberti, C.Chen, E.Giguet, J.Rullier, J.Wurtele and R. Temkin MIT in the Proceedings of this Meeting.

[6] "Initial Operation of a High Power K-Band, Harmonic Gyro-Klystron" J. Calame, H. Matthews, B. Hogan, M. Lee, J. Cheng, C. Striffler, V. Granatstein and $M$. Reiser $U$. Maryland in the Proceedings of this Meeting.

[7] "Gyroklystron Research for Application to TeV Linear Colliders" M. Reiser et. al in $X V^{\text {th }}$ International Conference on High Energy Accelerators, HEACC'92 Hamburg 1992,p. 924.

[8] "The NRL X-band Magnicon Amplifier" S.Gold, C. Sullivan and W. Manheimer and B. Hafizi in the Proceedings of this Meeting.

[9] "First Test of the X-band Pulsed Magnicon", O. Nezhevenko et. al. in the Proceedings of this Meeting.

[10] "Two Stage, High Power X-band Amplifier Experiment", E. Kuang, G.S. Kerslick, J.D. Ivers, J.A. Nation and L. Schächter; in the Proceedings of this Meeting.

[11] "The FEL as a power source for a high gradient accelerating structure" A.M. Sessler, AIP Conf. Proc. 91 p.154 (1982).

[12] "High Efficiency extraction microwave radiation from a tapered wiggler FEL" T.J. Orzechowsky et. al. Phys. Rev. Lett. 57, 2172(1986).

[13] "Two-beam linear colliders" by K. Hubner in $X V^{\text {th }}$ International Conference on High Energy Accelerators, HEACC'92 Hamburg 1992,p. 791.

[14] "FEL as an RF source for the Linear Collider" A.A. Kaminsky, in $X V^{\text {th }}$ International Conference on High Energy Accelerators, HEACC'92 Hamburg 1992,p. 900.

[15] "14GHz VLEPP Klystron", V. Balakin, in Proc. of the $3^{\text {rd }}$ EPAC, Berlin, Germany 1992.

[16] "Narrow Band High Power Traveling Wave Amplifier", L.Schächter and J.A. Nation; The third Work- shop on Advanced Acceleration Concepts, Port Jefferson, Long Island, NY 14-20 June 1992.

[17] "Common Analysis of the Relativistic Klystronand the Standing Wave FEL" J.S. Wurtele, D.H. Whittum and A.M. Sessler in $X V^{\text {th }}$ International Conference on High Energy Accelerators, HEACC'92 Hamburg 1992,p. 508.

[18] "Beam-wave interaction in quasi-periodic structures" L. Schächter and J.A. Nation see the Proceedings of this Meeting. 